\title{
Designing Smart Homes for Dependent Persons Assistance
}

\author{
Rim Jouini, Karima Maalaoui and Leila Azouz Saidane \\ Ecole Nationale des Sciences de l'Informatique, University of Manouba Campus of \\ Manouba, Manouba, Tunisia
}

\author{
Received 23 February 2014; Accepted 24 May 2014 \\ Publication 4 August 2014
}

\begin{abstract}
Evolution of technology has permitted to help dependent persons by implementing new systems to assist them at their homes. The majority of these systems are based on Wireless Sensor Networks (WSN) using 802.15.4 standard at the MAC level. In this paper, we propose a solution to permit a continuous assistance for dependent persons, called Domotic Assistance for Dependent Persons (DADP). We also propose a new MAC protocol to enhance QoS requirements, especially for alarm management in critical situations. Simulation results have shown that our proposed solution performs better than 802.15 .4 in terms of reliability, energy consumption and loss ratio.
\end{abstract}

Keywords: WSN, IEEE 802.15.4.

\section{Introduction}

Recent years has known a continuous increase in the number of dependent persons. A more simple and transparent hardware, attentive and adaptable to the user lifestyle, is still an open issue [11]. To deal with this issue, we propose an integrated and low-cost wireless architecture for noninvasive monitoring. WSN is an example of a technological solution adopted by researchers to go toward the idea of ubiquitous computing and smart

Journal of Machine to Machine Communications, Vol. 1, 161-176.

doi: 10.13052/jmmc2246-137X.125

(c) 2014 River Publishers. All rights reserved. 
environment [11]. These networks are formed by a set of miniature nodes that collect information and send it to a configured sink node that forwards it to a monitoring system. A sensor node is composed by a sensing unit for acquiring data, a micro-controller for data computation, a wireless communication unit and a limited power battery. WSN will permit a continuous assistance for dependent people in their homes as opening doors and turning off or on the light automatically. This needs real time traffic to respond to dependent persons needs in a real time manner. In the MAC level, IEEE 802.15.4 standard is considered as the most widely adopted solution for WSNs $[1,2]$. However, this standard cannot support application with QoS requirements and is not suitable for real time traffic management [3]. To solve this problem, we have defined a new MAC protocol under LEACH. This new protocol will enhance QoS requirements especially for alarm management in critical situations by providing them with high priority while ensuring low energy consumption and high reliability 13 The rest of the paper is organized as follows. The first section gives an overview of the IEEE 802.15.4 protocol. The second section presents our solution, called DADP, for assisting dependent persons in their homes. Performance evaluation of DADP is presented in the third section. Finally, we conclude the paper and outline our future work.

\section{IEEE 802.15.4 Protocol}

The following section gives a brief overview of IEEE 802.15.4 MAC protocol. This protocol is defined to provide applications with low throughput and latency requirements. Besides, low complexity, low cost, low power consumption, low data rate transmissions are the key characteristics of its adoption for WSNs [2]. IEEE 802.15.4 supports three topologies: star, tree or mesh topology. Two types of devices can coexist: FFD (Full-Function Devices) which implement all the functions of the standard and RFD (Reduced-Function Devices).

IEEE 802.15.4 MAC has two operational modes: a beacon enabled mode and a non-beacon enabled mode. In the first mode, the network is controlled by a coordinator, which regularly transmits beacons for device synchronization and association control. The channel is bounded by a superframe structure as illustrated in Figure 2. The superframe consists of both active and inactive periods. The active period contains three components: a beacon, a Contention Access Period (CAP) and a Contention Free Period (CFP). The coordinator interacts with nodes during the active period and sleeps during inactive period. There are a maximum seven Guaranteed Time Slots 


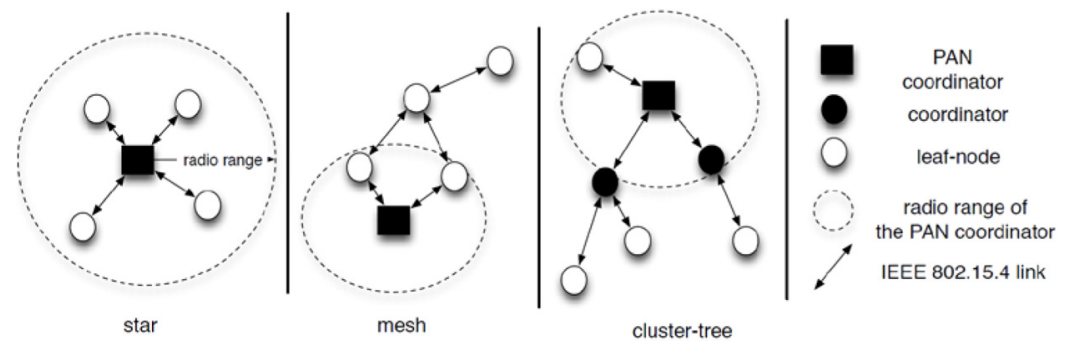

Figure 1 The different topologies proposed in IEEE 802.15.4

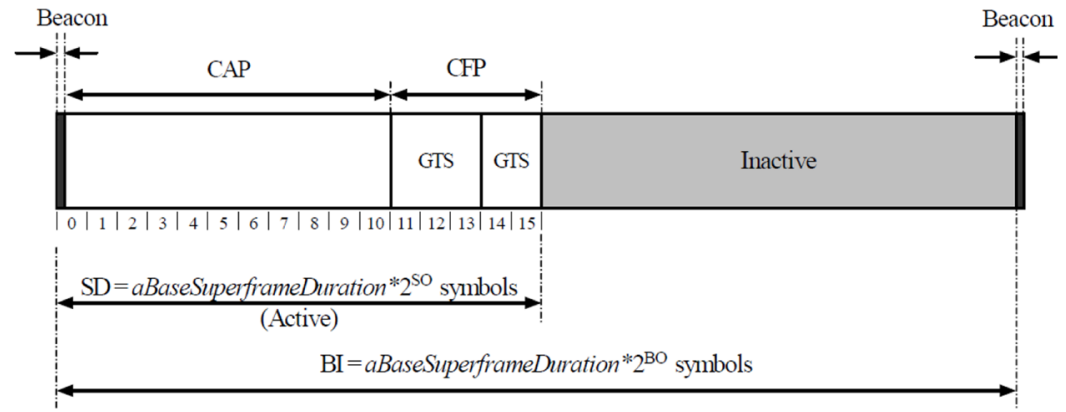

Figure 2 IEEE Superframe [4]

(GTS) in the CFP period to support time critical traffic. In the beaconenabled mode, a slotted CSMA/CA protocol is used in the CAP period while in the non-beacon enabled mode, unslotted CSMA/CA protocol is used [4].

\section{Related Works}

An aging population, combined with sedentary lifestyle and poor diet, is resulting in an increasing number of dependent people that require help. Having a person as a helper at home is very expensive. Wireless sensor network technologies have the potential to offer large-scale and cost effective solutions to this problem. They permit in-home monitoring of elderly, patients or disabled persons. Such technologies have enabled remote monitoring of dependent persons in their own homes or environment and improved care provider ability to deliver help.

WSN have to send periodic message to a monitoring center by conducting their messages to a specified access point. Abnormal critical situation 
cases should have a special treatment to insure timely delivery for efficient intervention.

Following the messages they have to undertake, two types of traffic can be found in a WSN:

- Periodic traffic indicates the best function of node

- Alarms generated in critical situations.

These messages need real time and high priority treatment.

In the literature, several solutions have been proposed for dependent persons assistance [10] as GRADIEN, PROSAFE, EVIENT, ERGDOM and AILISA. Next, we will focus only on the two first solutions as they are the most important of all solutions. GRDEIEN was developed by INSERM U558 and ProSafe system was developed by LAAS in Toulouse, to monitor the nocturnal wanderings in a hospital room, called Hospital Smart Rooms. GARDIEN aimed to trigger an alarm if the patient quits the room and help diagnose symptoms of a disease through the study of motor behavior and appreciate the effects of treatment on motor behavior of the patient. PROSAFE permits also the same type of remote monitoring of frail patients, but it improves the previous system using wireless sensors, but it proposes a system of automatic alarm triggering based on various criteria.

These different projects were developed for dependent person aim, especially for monitoring health. Our idea is to look for this dependent person

from another sight that consists to assist this person in their own homes or environment to facilitate their activities and improved care providers ability to deliver help in critical situations.

\section{Idea of Smart Home}

Smart home integrates information and communication-technology, whereby the different components communicate via a local network. This network communicates with the external world by telephone or through the Internet, which means that the smart home can be programmed from inside or outside the house.

Smart home technology can contribute to increased independence and safety for disabled person. Safety is the biggest advantage of smart home technology. Smart home is advertised to call customers as a means of living more comfortably in their home. To the disabled person, this advantage means an increased ability to cope with the activities of daily living, resulting in increased independence. 


\section{DADP Solution}

We propose a novel solution called Domotic Assistance for Dependent Person (DADP) and a new medium access control protocol to manage alarms and periodic messages. DADP will permit assistance for dependent persons in their habitat as shown in Figure 3.

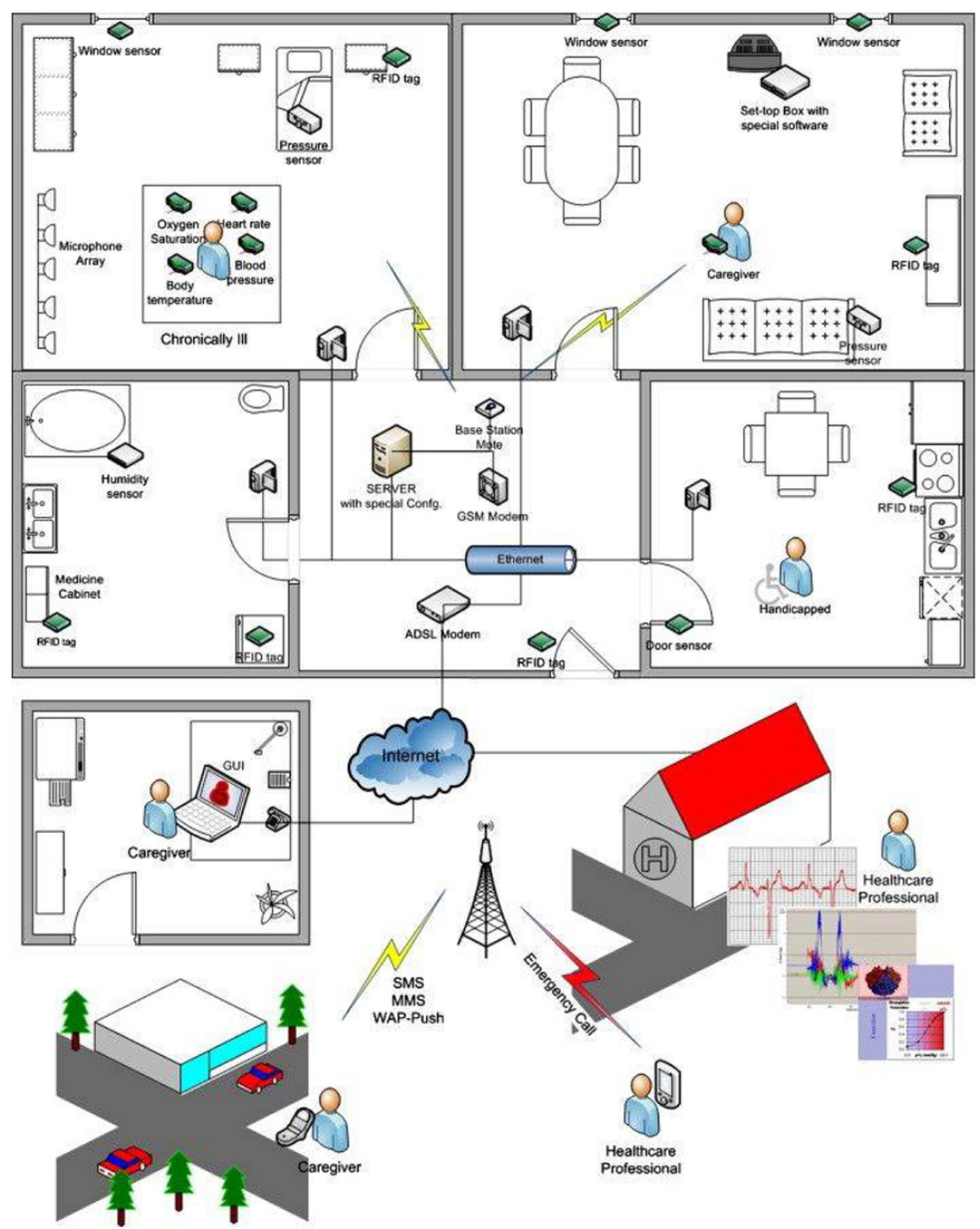

Figure 3 WSN overview in a sample smart home for person assistance [5] 


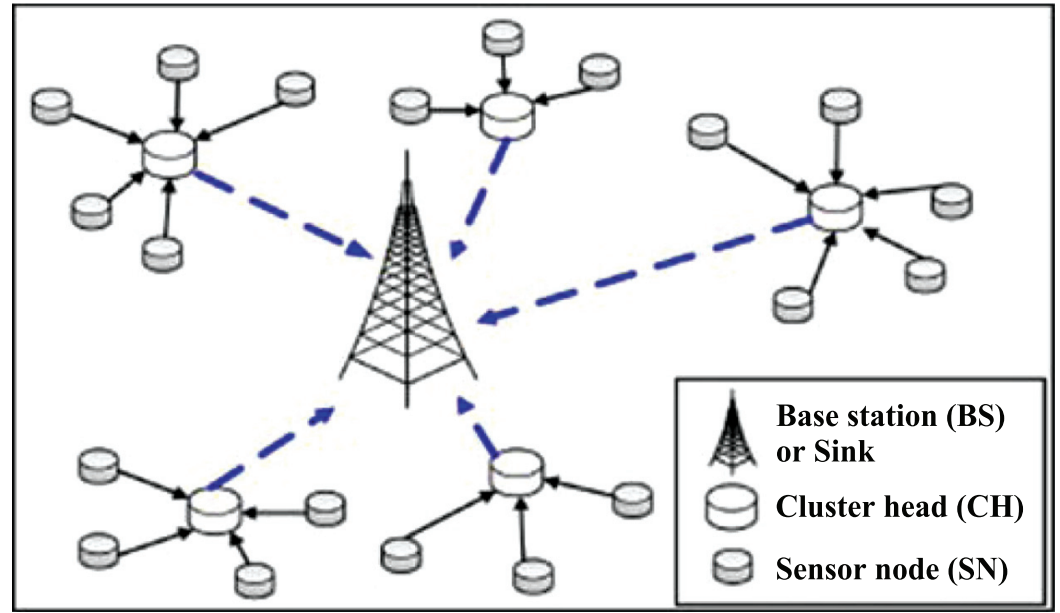

Figure 4 Cluster-based WSN [6]

To ensure the safety of a dependent person, we place, in each room, a gas detection sensor node and a smoke detection sensor node. These sensors generate alarms that are transmitted to the center monitoring or referred person. The actuators nodes are also placed in every room to manage brightness, window pane and temperature as shown in Figure 2. Actuators of our DADP solution are:

- Gas sensor nodes.

- Smoke sensor nodes.

- Light sensor nodes.

- Temperature sensor nodes.

These elements generate a periodic traffic and alarms. The periodic traffic permits to verify the good function of the sensor nodes in every room. This traffic is received by a room coordinator sensor (Room head) and will be relayed to the Sink. The Alarms are generated upon detection of smoke or gases and it should be sent immediately in a short time to have an early intervention.

All sensor and actuator nodes form a communication network in the smart home. This network can be organized in two topologies: a Cluster-based WSN topology (CWSN) or a Flat-based WSN topology (FWSN).

In FWSN, the multi-hop communication generates an important flow of information and is high level energy consuming. Thus, the control traffic generates a high overhead in the detriment of the effective communication traffic [6]. To solve this problem, one solution could be dividing the network into clusters. 


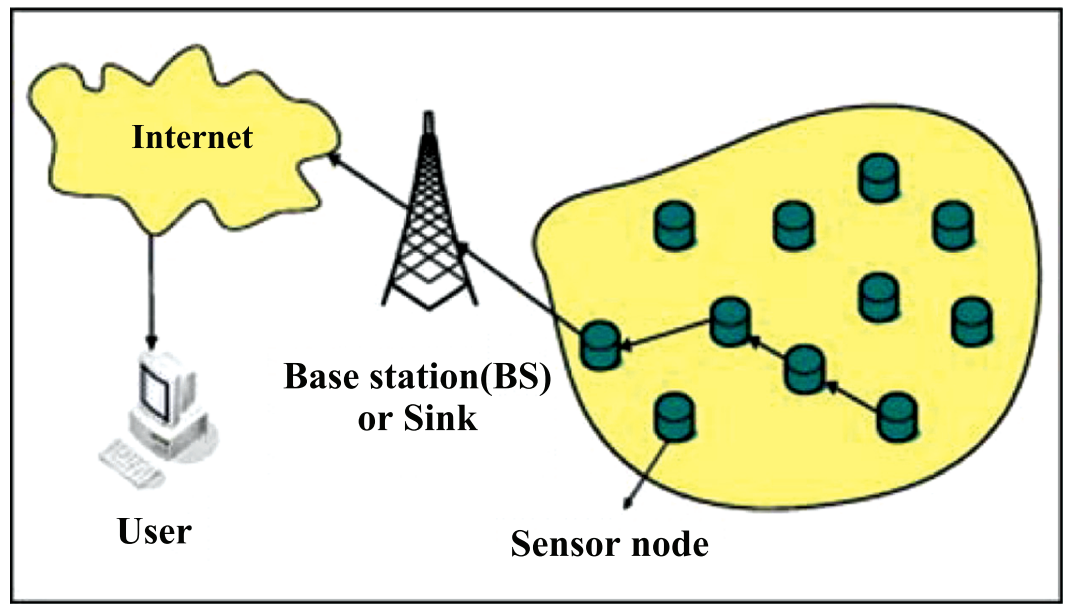

Figure 5 Flat-based WSN [6]

For CWSN, geographically nearby sensor nodes form a cluster identified by a particular node among them called cluster-head $(\mathrm{CH})$. The $\mathrm{CH}$ manages its cluster and aggregates the traffic inside the cluster before sending it to the sink. Thus, both overhead and energy consumption are reduced. Many protocols as CWSN have been proposed as LEACH APTEEN and Pegasus [6].

To organize the wireless sensor network in the home of a dependent person, we adopt the cluster star architecture by using the protocol LEACH. We propose a new access method to overcome the limitations of the access standard IEEE 802.15.4 MAC layer. This method will be used to enhance the management of alarms and periodic messages. It relies on the static nature of the home network to implement a fixed scheduling strategy. It will give to sensors reporting urgent information a higher priority by delaying transmission of packets that are reporting periodic low priority messages. The proposed MAC protocol be used with LEACH protocol.

In the same context, Hanen and al. [7] have proposed a novel medium access control protocol for communication in a WBAN for remote monitoring of physiological signals of patients by implementing a fixed scheduling strategy in which they keep the same concept of beaconing as used in IEEE 802.15.4 but they change the decomposition of the superframe that they called E-Health Access Channel Mechanism (EHACM).

They divide the E-HACM superframe into equal Allocated Time Slots (ATSs) (see Figure 6). Each ATS is allocated to a node according to a static 


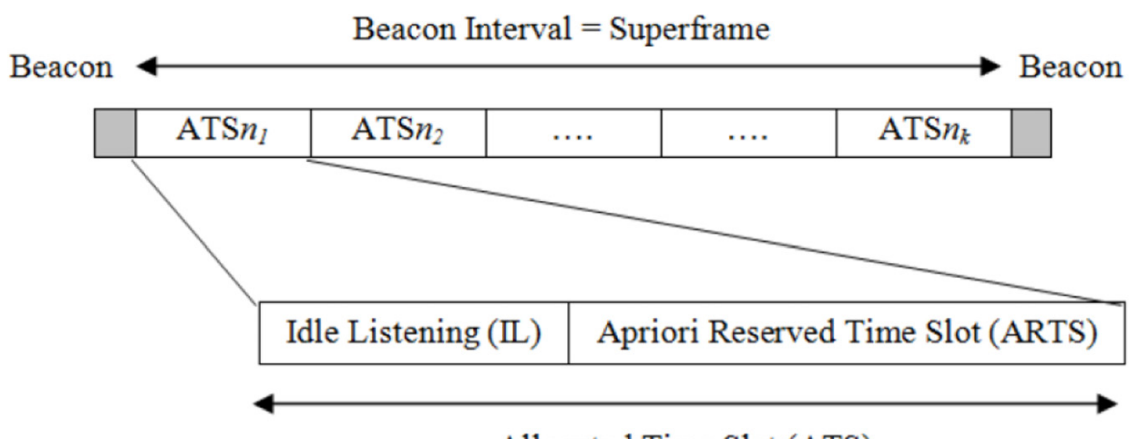

Allocated Time Slot (ATS)

Figure 6 E-HACM Superframe [7]

predefined scheduling. Each node can be in sleep mode and wakes up only during its allocated ATS or if it has an alarm. An ATS contains two sub periods: Idle Listening (IL) and Allocated Reserved Time Slot (ARTS). IL is dedicated for sending alarms if any node of the network needs to, while the ARTS period is reserved to the owner of the current ATS to send its periodic message if no alarm was generated during the IL period of the current ATS. The owner of the current ATS has to listen during IL and cancel its periodic sending if another node has generated an alarm during the IL period. Once a node has completed its sending, it returns into sleep mode until the next superframe.

In our solution DADP, the MAC protocol (Figure 7) is inspired from E-HACM. In addition, the IEEE 802.15.4 superframe is divided into 16 slots, each one is assigned to a node. Our contribution is limited only to MAC layer using the IEEE 802.15.4 default physical layer. That's why we limit ourselves to 16 slots in our solution. The general idea is to divide the superframe into equal Cluster Allocated Time Slots (ATSs-Cluster) (Figure 3). Each ATS-Cluster is divided into equal ATS-Node according to the E-HACM method. Each node can be in sleep mode and wakes up only during its allocated ATSNode or if it has an alarm. An ATS-Node contains two sub periods: Idle Listening (IL) and Allocated Reserved Time Slot Node (ARTS-Node). IL is dedicated for sending alarms if any node of the network needs to, while the ARTS-Node period is reserved to the owner of the current ATS-Node to send its periodic message if no alarm was generated during the IL period of the current ATS-Node. The owner of the current ATS-Node has to listen during IL and cancel its periodic sending if another node has generated an alarm during 


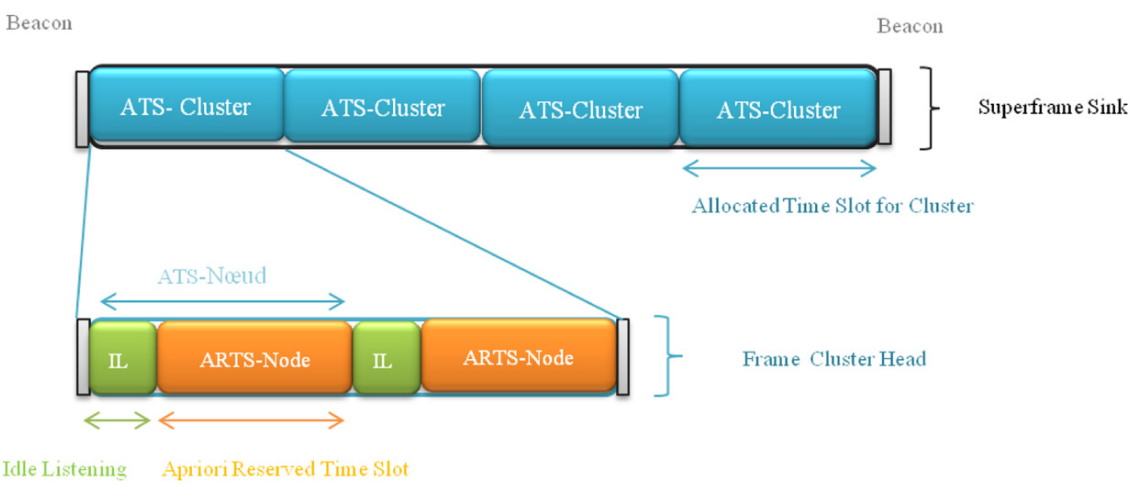

Figure 7 DADP Superframe

the IL period. Once a node has completed its sending, it returns into sleep mode until the next superframe.

\section{Performance Evaluation}

We implemented DADP in Castalia [8] under OMNET++ simulator [9]. We have evaluated the performances of DADP according to the following criteria:

- The MAC level packet breakdown

- The number of packets received per sink

- The consumed energy

- The loss ratio

We run the same simulation scenarios for IEEE 802.15.4 with its two modes (Beacon and Beaconless modes) in order to compare them with the performances of our proposed solution while varying the rate. Simulation results are shown in the following subsections.

A. MAC level packet breakdown

Figure 8 shows the distribution of packets at the MAC level "break Packet" while varying rate. We notice no packets failure because access is based on reserved slots.

B. Number of packet received

For IEEE 802.15 .4 beacon mode, the number of packet received by sink is limited because we have a maximum of 7 GTSs in an IEEE 802.15.4 superframe. Thus, some nodes will cancel their messages since they will not have allocated GTS. 
170 Rim Jouini et al.
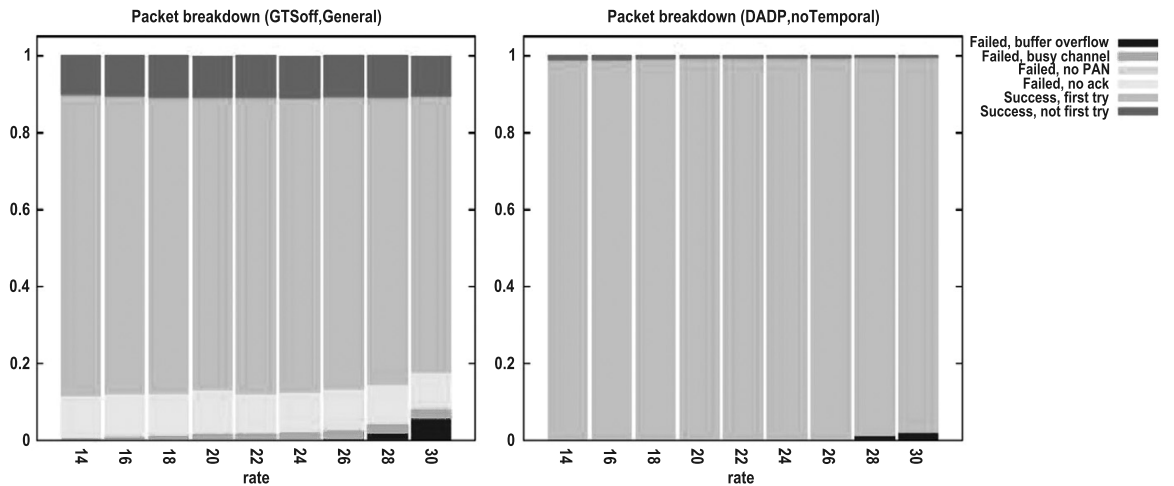

Figure 8 MAC level packet breakdown

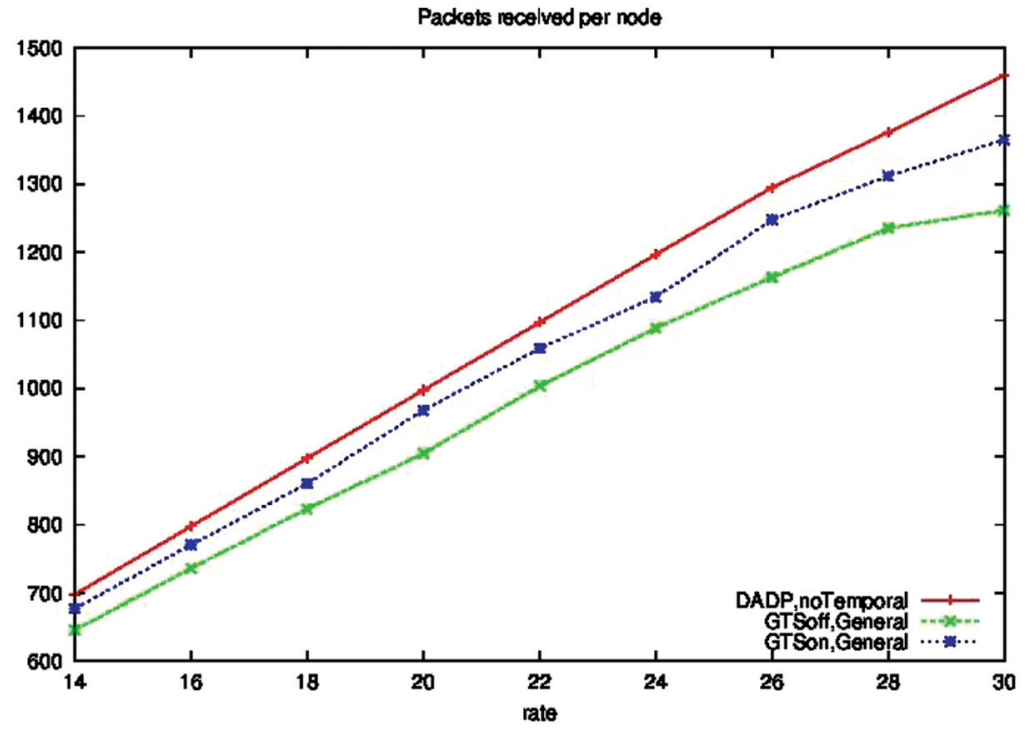

Figure 9 Number packets received per sink

\section{Energy consumption}

Figure 10 shows energy consumption while varying rate. We notice that, although the values of the two protocols are almost the same, DADP is more energy efficient compared to IEEE 802.15.4 due to the static scheduling defined in DADP. Nodes spend more time in sleep state, thus conserving their energy. The measures obtained by GTSoff can be explained due to use of the method CSMA/CA. 


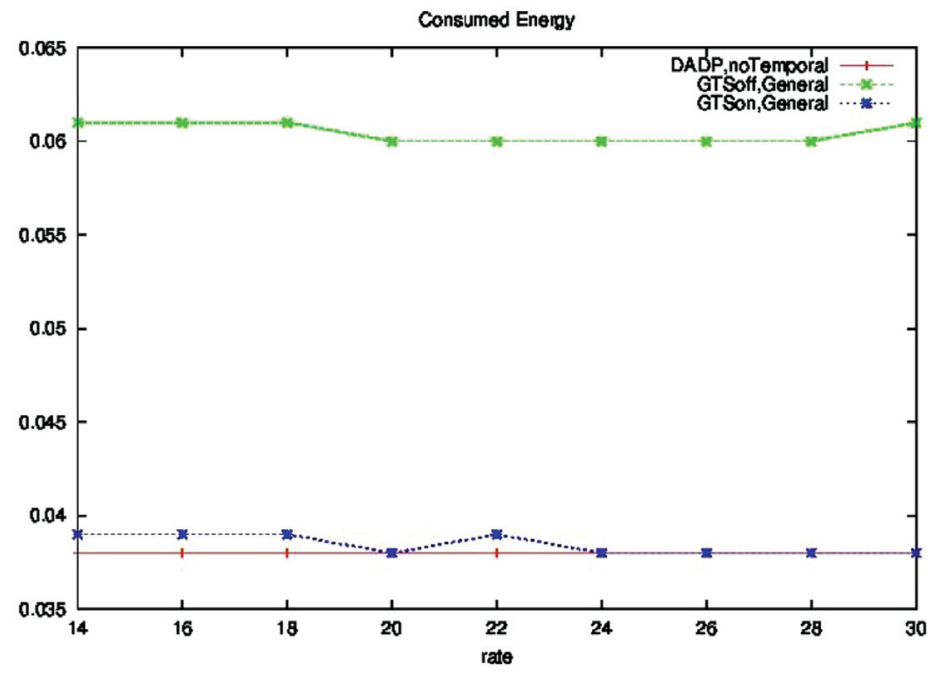

Figure 10 Consumed energy

D. Loss ratio

Figure 11 shows the loss ratio generated by DADP. We notice that the majority of packets are successfully sent for the first try. Therefore, DADP is more suitable for a real time transfer and is more reliable for alarms management.

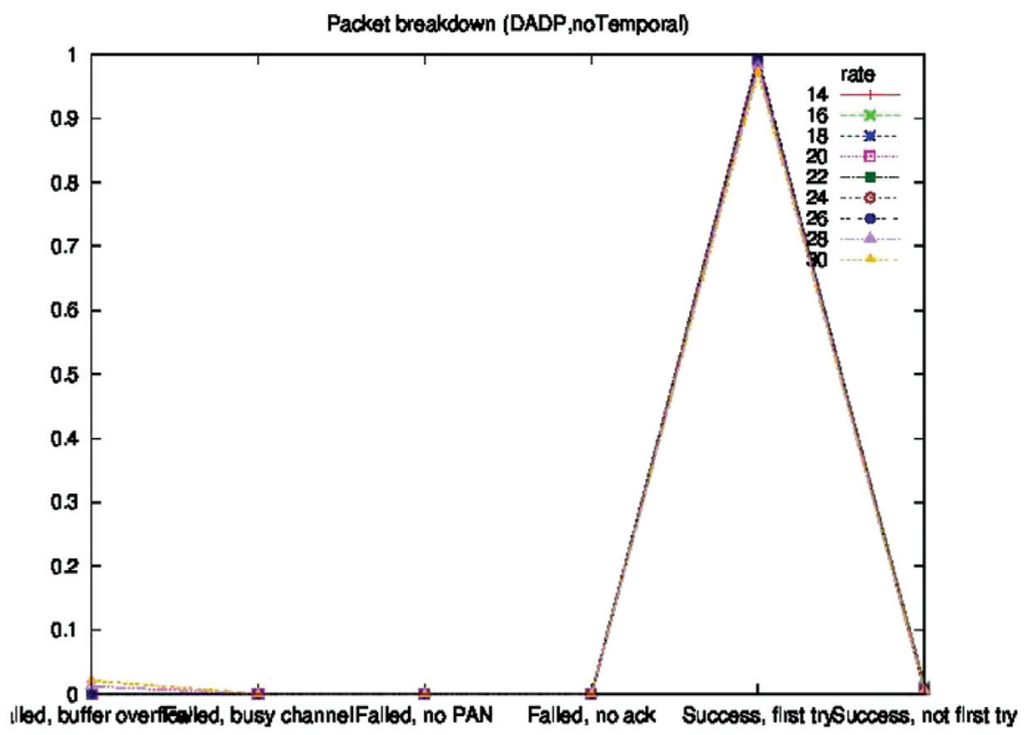

Figure 11 Loss ratio 


\section{Rim Jouini et al.}

\section{Conclusion}

In this paper, we proposed a WSN based solution for dependent persons assistance at their smart homes. This solution, called DADP, permits to a distant manager to take care of a dependent person at his smart home by communicating with the WSN. We have also introduced a novel MAC protocol to manage alarms and periodic messages generated by sensors. This protocol relies on the static nature of the home network to implement a fixed scheduling strategy. The new MAC protocol insures high reliability for critical situations.

DADP is based on a fixed scheduling mechanism where each sensor has a pre-assigned time slot, called ATS-Node, during an ATS-Cluster. Each node should be awake only during its preassigned ATS-Node. Nonetheless, in case of critical situations, a sensor should generate immediately an alarm. It can use any ATS-Node of ATS-Cluster for its alarm message transmission. To avoid collisions, each ATS-Node begins with an idle listening sub period, during which any sensor handling an alarm message can transmit immediately, while the assigned node has to listen before deciding to cancel or to send its periodic message.

Our solution has been validated through simulations using Castalia and OMNET++ simulator. We have proved that the proposed solution enhances reliability transmission, energy consumption and loss ratio compared to IEEE 802.15.4.

In future works, we plan to extend our solution by adding a WBAN as dynamic cluster to the DADP static clusters. Modeling the behavior of the dependent person in its habitat using Markov chain for more efficient consumption energy may be a second interesting future work.

\section{References}

[1] B. Zhen, Li. H-B and R. Korno. IEEE Body Area Networks for Medical Applications. The International symposium on Wireless Communication Systems (ISWCS 2007), pp. 327-331, Norway, October 2007.

[2] P. Kumar, M. Gunes, A.B. Almamou and J. Schiller. Real-time, Band width and Energy Efficient IEEE 802.15.4 for Medical Applications. 7th GI/ITG kuVS Fachgesprach Drahtlose Sensornetze, FU Berlin, Germany, September 2008.

[3] F. Chen, T. Talanis, R. German, F. Dressler. Real-time enabled IEEE 802.15.4 sensor networks in industrial automation. IEEE International Symposium on Industrial Embedded Systems (SIES'09), pp. 136-139, Lausanne, Switzerland, July 2009. 
[4] S. Ullah, H. Higgins, B. Braem, B. Latr, C. Blondia, I. Moerman, S.Saleem, Z. Rahman, and K.S. Kwak, "A Comprehensive Survey of Wireless Body Area Networks - On PHY, MAC, and Network Layers Solutions", Springer: Journal of Medical Systems, pp.1065-1094, June 2012.

[5] H. Alemadr and C. Erosy, "Wireless Sensor Networks for Healthcare: A Survey" ScienceDirect: Journal of Computer Networks, Volume 54, Issue 15, pp.2688-2710, October 2010.

[6] Shun-Sheng Wang, Kuo-Qin Yan, Shu-Ching Wang, Chia-Wei. An Integrated Intrusion Detection System for Cluster-based Wireless Sensor Networks. Journal Expert Systems with Applications: An International Journal, Volume 38, Issue 12, pp. 15234-15243, November, 2011.

[7] Hanen Idoudi, Fatma Somaa, Leila Azouz Saidane. "Alarms Management in Wireless Body Area Networks". International Conference on Computer Systems and Applications (AICCSA), 2013 ACS, pp. 1-4, France, May 2013.

[8] Castalia simulator for Wireless Sensor Networks (WSN), Body Area Networks(BAN) and generally networks of low-power embedded devices. http://castalia.research.nicta.com.au/index.php/en/. Last access on June 2013.

[9] OMNET++ Network Simulator. http://www.omnetpp.org/. Last access on June 2013.

[10] M. Chan, E. Campo, D. Estve, PROSAFE, a multisensory remote monitoring system for the elderly or the handicapped in Independent Living for Persons with Disabilities and Elderly People, IOS Press, 1st International Conference On Smart homes and health Telematics (ICOST'2003), Sept. 24 26, 2003, pp. 89-95, Paris France.

[11] M. R. Alam, M. B. I. Reaz, and M. Ali, "A review of smart homes Past, present, an future," IEEE Trans. Syst. Man Cybern. C, Appl. Rev., vol. 42, no. 6, pp. 1190-1203, Nov. 2012. 


\section{Rim Jouini et al.}

\section{Biographies}

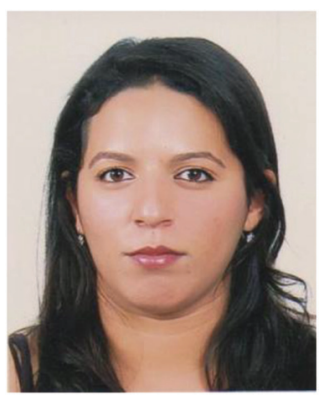

Rim JOUINI member and researcher of CRISTAL - ENSI lab Tunisia, received the diploma of national computer engineering from ENSI, University of Manouba Tunisia in 2013, and MS degree from ESTI, University of Carthage Tunisia in 2010 and bachelor degree in Computing from ISET of RADES 2008.

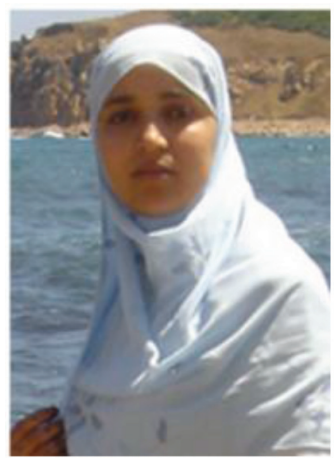

Karima MAALAOUI is an assistant professor at Carthage University's Faculty of Sciences. She received the Engineer degree in 2003 and The $\mathrm{Ph}$. D. in 2009, all from the National School of computer sciences-university of Manouba. Her areas of interest and research include QoS and security in wireless and mobile networks, M2M communications, Internet of things and Wireless sensors networks.

Leila Azouz Saïdane is Professor at the National School of Computer Science (ENSI), at The University of Manouba, in Tunisia and the Chairperson of the PhD Commission at ENSI. She was the Director of this school and the 
supervisor of the Master's Degree program in Networks and Multimedia Systems. She is the co-director of RAMSIS group of CRISTAL Research Laboratory (Center of Research in Network and System Architecture, Multimedia and Image Processing) at ENSI. She collaborated on several international projects. She is author and co-author of several papers in refereed journals, magazines and international conferences. 
